\title{
Commentary: It's all about the distal
}

\author{
Anthony L. Estrera, MD
}

From the Department of Cardiothoracic and Vascular Surgery, McGovern Medical School at UTHealth, Houston, Tex.

Disclosures: Author has nothing to disclose with regard to commercial support. The Department of Cardiothoracic and Vascular Surgery has received research support from Terumo Aortic.

Received for publication Jan 16, 2019; accepted for publication Jan 16, 2019; available ahead of print Feb 20, 2019.

Address for reprints: Anthony L. Estrera, MD, Department of Cardiothoracic and Vascular Surgery, McGovern Medical School at UTHealth, 6400 Fannin St, Suite 2850, Houston, TX 77030 (E-mail: anthony.L.Estrera@ uth.tmc.edu)

J Thorac Cardiovasc Surg 2019;157:e235-6 $0022-5223 / \$ 36.00$

Copyright (c) 2019 by The American Association for Thoracic Surgery https://doi.org/10.1016/j.jtcvs.2019.01.065

In this issue of the Journal, Urbanski ${ }^{1}$ reports his approach to the reconstruction of the transverse aortic arch with a multibranched Dacron polyester fabric graft. Acknowledging that as many as $45 \%$ of patients undergoing total transverse arch replacement may require a distal thoracic aortic intervention, ${ }^{2}$ Urbanski ${ }^{1}$ modified the arrangement of supra-aortic bypasses to be performed. As illustrated in his report, ${ }^{1}$ the predesignated cannulation branch of the 4-branched graft is instead used for the bypass to the left subclavian artery (LSCA), and the predesignated LSCA branch is instead used for arterial cannulation. As with current commercially available multibranch Dacron polyester fabric aortic grafts in the United States, this configuration-with cannulation branch anterior and proximal to the most distal branch-may provide an increased landing zone $(1.5 \mathrm{~cm})$ for subsequent thoracic endovascular interventions (Figure 1). By using this supra-aortic reconstruction, Urbanski ${ }^{1}$ lengthens the landing zone for future thoracic endovascular aortic repair without altering his general arch reconstruction.

To lengthen the landing zone during total arch replacement without use of the elephant trunk, others have proposed the "zone 2" arch replacement. ${ }^{3}$ Here, the distal anastomosis is performed at the zone 2 level and the left common carotid and innominate arteries are debranched with grafts originating from the ascending aortic graft. This approach moves the landing zone proximally into the ascending aortic graft, affording a technically easier distal anastomosis, because it is now more anterior, which also potentially reduces the incidence of recurrent laryngeal nerve injury. As appropriately noted by Urbanski, ${ }^{1}$ the zone 2 arch approach requires longer bypasses, which raises concerns of graft kinking and decreased late patency. In addition, when reoperative cardiac surgery is required after previous zone 2 arch repair, the supra-aortic bypasses during the redo sternotomy may be an issue.

When dealing with transverse arch replacement, a technical concern is always the distal anastomosis. Although

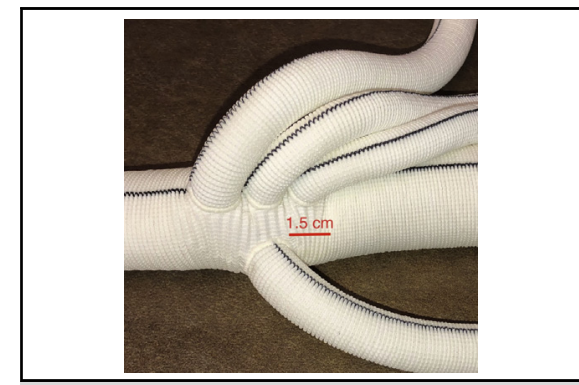

US commercially available Dacron transverse aortic arch graft with multiple branches.

\section{Central Message}

Urbanski is to be commended for a new way to address the distal anastomosis in transverse arch replacement. It's all about the distal.

See Article page e229.

the technique described may be of less significance as the use of the frozen elephant trunk expands, Urbanski ${ }^{1}$ is to be commended for providing an interesting way to address the distal anastomosis, and facilitate a subsequent distal

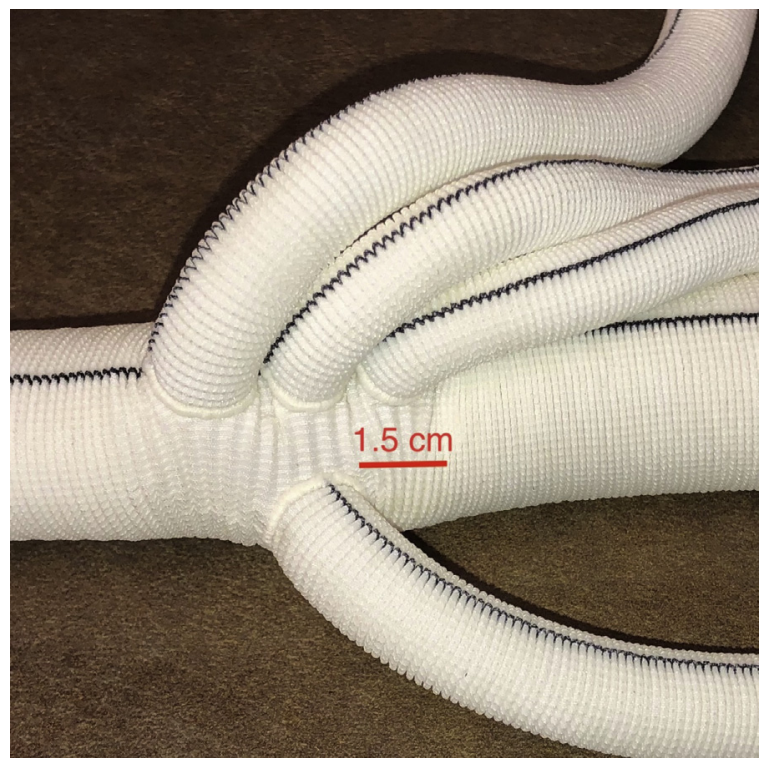

FIGURE 1. US commercially available Dacron polyester fabric transverse aortic arch graft with multiple branches. The designated cannulation branch is positioned anteriorly and $1.5 \mathrm{~cm}$ more proximally to the most distal margin of the designated left subclavian artery branch. 
intervention. Multiple considerations bear mention before performing the distal anastomosis.

1. Is the proximal descending thoracic aorta involved? If so, does it need to be addressed immediately, or can it be delayed? As the frozen elephant trunk prosthesis becomes more widely accepted and available in the United States, it likely has become the favored approach, because it immediately addresses the proximal descending thoracic aorta and provides the optimal landing zone for subsequent endovascular procedures. The approach described in this report, like the zone 2 approach, provides landing zones for subsequent interventions; however, the frozen elephant trunk may eventually be more applicable.

2. How deep is the patient's chest? If the chest is deep $(>10 \mathrm{~cm}$ from anterior sternum to LSCA), then performing the distal anastomosis at the LSCA is challenging. A zone 2 arch repair mitigates this issue; however, the longer debranched bypasses-as well as addressing the LSCA—become an issue.
3. Is the LSCA revascularized at the time of the arch replacement, or at a later time by left carotid to subclavian artery bypass or branched thoracic endovascular aortic repair? What are the statuses of the left vertebral artery and posterior cerebral circulation? If the left vertebral artery is dominant, when should we revascularize the LSCA?

In the end, not only does this report of Urbanski ${ }^{1}$ highlight issues to consider when performing transverse arch replacement but it also ultimately emphasizes that it's all about the distal.

\section{References}

1. Urbanski PP. Prospective planning during conventional aortic arch replacement: the role of landing zone preparation for future stent grafting. J Thorac Cardiovasc Surg. 2019;157:e229-34.

2. Estrera AL, Sandhu HK, Miller CC, Charlton-Ouw KM, Nguyen TC, Afifi RO, et al. Repair of extensive aortic aneurysms: a single-center experience using the elephant trunk technique over 20 years. Ann Surg. 2014;260:510-6; discussion 517-8.

3. Desai ND, Hoedt A, Wang G, Szeto WY, Vallabhajosyula P, Reinke M, et al. Simplifying aortic arch surgery: open zone 2 arch with single branched thoracic endovascular aortic repair completion. Ann Cardiothorac Surg. 2018;7:351-6. 